УДК 372.881 .1

DOI 10.25205/1818-7919-2018-17-4-132-138

\author{
王中俊 \\ 新西伯利亚国立大学 \\ 630090 俄罗斯新西伯利亚市皮罗果夫街 1 号 \\ mex2011@126.com \\ 对外汉语初级阶段语法教学微技能
}

本文从实际课堂教学的微观层面出发, 结合分析初级阶段易出现的语法偏误, 从语法点的 导入、解释、操练、归纳、练习、复习等环节来探讨语法教授的微技能。根据不同语法项目 的特点和紧扣初级汉语语法教学实际情况, 本着少用术语、精讲多练采用语言对比法、简化 浅化法、语义语用分析法、结构法、情境法等教学方法。

关键词: 对外汉语、初级、语法教学、微技能。

\title{
引言
}

对外汉语教学基本目的就是要教给汉语学习者运用汉语进行社会交际的能力, 而汉语语法 作为汉语的四大要素之一, 是汉语词、短语、句子乃至篇章的组织规则, 是构成汉语学习者 进行汉语交际能力的核心因素之一。俄罗斯学生在语法学习过程中的语法偏误也主要表现在 虚词和语序。经过对俄罗斯学生在语法学习、平时作业以及期末考试文章中出现的偏误进行 总结, 可以看到俄罗斯学生语法偏误主要表现在遗漏、误加、误代、错序四大类。赵金铭指 出, 我们需要对外汉语教学语法, 而不是理论与方法。我们对外汉语教材上的语法通常既要 兼顾语法点解释的严谨性又要讲求实用性, 简洁明了, 便于学习。赵金铭指出, 对外汉语语 法教学的总体目标是要培养外国学生用汉语组词造句、连句成篇的表达能力。习得者在学习 汉语的过程中首先得解决正误问题 [赵金铭, 2003]。所以, 很有必要从教材语法点和学生易 出现的偏误入手来探究如何提高语法教学效率。

本文涉及到的语法点均来自本人实际上课使用过的综合课课本《新实用汉语课课本》（第 一、二册）和口语课课本《汉语会话 301 句》 (上、下册) 两套教材。两套教材都有语法注

Ван Чжунизюнь. Микрометоды в обучении грамматике китайского языка как иностранного на начальном этапе // Вестн. НГУ. Серия: История, филология. 2018. Т. 17, № 4: Востоковедение. С. 132-138. (на кит. яз.) 
释讲解和相应的配套练习。其中《新实用汉语课本》综合课课本, 前两册共有语法点 106 项 （包括注释语法）, 且讲解相对详细、突出结构; 《汉语会话 301 句》是口语性的教材, 上、 下两册共有语法点 71 项, 且相对简略。《新实用汉语课本》教材中语法练习主要通过情景对 话来练习, 有配套练习册; 《汉语会话 301 句》教材中语法练习主要通过各类填空、改写句 子、替换练习等, 无配套练习册。

课堂上语法讲解步骤分为语法点导入、操练、归纳、练习、课后作业这样一个教学环节 [李 珠、蒋丽萍 2008]。本文从语法点的导入、解释、操练、归纳、练习、复习等环节来探讨语法 教授的微技能。 。语法教学可采用的方法有很多种, 本文主要根据不同语法项目的特点和从学 习者实际情况出发, 本着少用术语、精讲多练采用语言对比法、简化浅化法、语义语用分析 法、结构法、情境法等。下面我通过教学案例的形式来展示以上教学法。

\section{一. 语言对比法}

教学中, 我注意到俄罗斯学生在使用汉语时尽量减少或者规避自己不熟悉或者不会的语法 项目, 而且习惯于套用俄语语法。将两种语言的语法进行对比, 可以快速有效地提升教学效 率。语言对比可以分为两类, 一是汉外对比, 一类是汉语内部对比。俄罗斯学生在学习使用 汉语时自觉不自觉地在将汉语与俄语进行对比。通常汉俄语法规则相同时常常出现语言正迁 移, 有利于学生学习, 比如语法汉语里的“动词谓语句”的一个结构“主语十动词十宾语”, 如“我 吃面包” ( Я ем хлеб.) , 这一语法与俄语相应表达习惯一致, 所以学生很容易掌握。反之, 汉语里有的语法点俄语里没有, 抑或有一些细微差别, 这样俄罗斯学生通常套用俄语表达习 惯, 则出现偏误。比如, “把”字句, 初级阶段俄罗斯学生学习后, 大多规避使用这一语法, 出现了该用而不用的现象, 如: “*我放书在桌子上了。” (Я положил книгу на стол.) 。结构 助词“得”, 俄语中没有相对应的表达, 学生常出现偏误, 如“*她汉语写很好看。”汉语的介词 和俄语的前置词也有诸多不同之处, 俄罗斯学生也常出现偏误, 如“*已经 12 点了, 他还在床。”

(Уже 12:00, он еще в кровати.)

此外, 汉语内部对比也是一种非常有效的方法。教学中我们发现初级阶段汉语学习者经常 泛化汉语语法规则出现了偏误。如“*你游泳了这个河”, “*猫没有会飞”, “*可是最近我很累 了”, *故事里的男主角叫贾宝玉, 是在有钱人的家里出生的, 分别是量词“个”、否定副词“没 有”、表变化助词“了”、句型“是......的”等语法点泛化的结果。

因此, 汉语老师在讲授语法时应该把两种语言进行对比强化正迁移, 通过教学减少负迁移, 同时注意学生在学习中将汉语语法过度泛化, 预测可能会出现的语法偏误, 并在教学中采取 一定的方法, 来提升教学效果。

\section{二. 简化浅化法}

俄语是一种形合语言, 而汉语是意合语言。因此, 汉语的语法系统性不是很强, 而且很多 汉语语法是根据语法自身的特点总结出来的, 条目繁杂, 规律性不是很强, 因而不太容易掌 握, 对于初学者更是如此。如助词“了”不仅可以分为“了 1 ”、“了 2 ”, 而且还在其他语法点中 常常出现, 如: 出现在“快/要……了”和可能补语 (吃得了/吃不了) 中, 而且这一语法点汉语 学习初期就出现了, 教材中通常将其分两到三次介绍。上述两个语法点内容十分繁杂, 学生

\footnotetext{
* Термину «微技能》в российской методике преподавания иностранных языков соответствует термин «микрометод» (см.: [Алещанова, Фролова, 2015]).
} 
经常敬而远之, 能不用则不用, 用则经常出错。如果不将上述语法点进行简化浅化, 初级阶 段学生就不会不知所措了。本人在听一些汉语老师课的时候, 发现有的汉语老师只是根据教 材的设计一点一点的给学生解释, 甚至有的时候, 老师自己也只是那么说那么用说不出所以 然，只是把内容讲完。我想这样效果肯定不佳。下面来谈谈我个人的做法。

关于助词“了”，我将其简单地概括为三个功能：表示变化、表示已经开始且不关注结果和 表示完成三种功能。

1、表示变化

天黑了; 我饿了; 听懂了; 明白了; 有工作了; 是老师了; 他来中国两年了。

2、表表示已经开始，不关注结果

他去上海了; 他回家了; 他去了上海了。

3、已经完成:

写了; 看了; 说了; 听了那首歌;

这样便可以简洁明了，让学生迅速掌握“了”的基本用法。此外，还有一点学生经常犯错的 地方就是否定副词和“了”连用的问题，如：*他没有去上海了。“没有”和“了”不能连用，可以 说: “他没有去上海。”或者“他不去上海了。”并把这两个句子的含义给学生讲清楚即可。当 然并不能完全解决问题, 但是初级阶段学生能掌握到这一程度并多加练习, 相信教学效果会 很明显。至于“了”其他用法和注意事项, 可以在中高级再进行讲解或者随文讲解即可。

\section{三. 语义、语用分析法}

“把”字句，教材《汉语会话 301 句》分为三次来介绍，其结构变体很多。关于“把”字句的 功能, 教材中这样界定：“把”字句常常用来强调说明动作对某事物如何处置以及处置的结果 [康玉华, 2006]。我发现很多学生在学过后还是不太理解这一语法功能。此外还有 7 个特点和 使用规定。关于这一语法点, 我看过一些示范课, 比如, 有一位老师上课老师引入这一语法 点通过很多动作，比如喝水、擦黑板等，先说主谓式的句子，然后再说“把”字句。学生当时 会用，但是我相信他们不会理解。“我喝水。”和“我把水喝了。”到底有什么差别。所以，这 一语法点老师很难解释出处置式的含义, 学生也很难理解。我将其浅化方式是通过设定一个 简单的问答场景来让学生理解, 效果很好。如有这样一个场景: 冰箱里有面包, 可是妈妈下 班后发现面包没有了，所以问：“面包呢？”，回答：“我把面包吃了。”而“我吃了面包。”则只 适合于回答“你吃什么了？”。至于“把”字句其他问题可以随文讲解即可。教学中这样引入这 一语法, 相信学生会知其然, 也知其所以然, 而且敢于使用。

\section{四. 结构法}

汉语的语法表现手段就是虚词和语序，所以很多汉语语法规则都是通过一定的句型结构来 展现, 初级阶段汉语语法教学也不例外。向学生展示语法结构, 学生能通过结构快速感知语 法的特征。适合于结构教学的语法点很多, 我个人教学中一般使用两种结构: 一是短语结构; 一是句子结构。

句子是由词和词组构成的。“词组”也可以叫做“结构”或“短语” [李德津、程美珍, 2008]。 短语结构可以视为汉语里一些词语具有固定的位置, 教学中我根据常见的一些语法语词进行 总结。如：“一点儿”和“有一点儿”。形容词/动词 + 一点儿、有一点 + 形容词/动词。如：贵一点 儿, 冷一点; 有一点儿贵、有一点儿冷。都/全/所有。关于两个以及以上个体+都: 我们都、 我和你都、大家都; 全+集体: 全国、全省、全市、全校、全班、全家; 所有 + 个体: 所有人、 
所有同学、所有花、所有学校、所有国家。汉语很多语法来自句型, 所以结构法在教学过程 中用的也较多。句子结构如：“是.......的”、存现句：地点+有/是 + 人/物、“比”字句：A 比 B+ 形容词/短语等特别适合使用。

\section{五. 情境法}

语法是词、短语、句子等语言单位的结构规律 [黄伯荣、廖序东, 2007]。学习语法知识不 是为了单纯的掌握语法知识, 最终目的还是要使语法称为一种正确表达汉语的能力。语境法 就是要把语法知识和实际语言运用结合起来。形成良好的汉语语感, 在汉语教学中强化语言 环境 [周健、陈群, 2011]。这样不仅可以将语法知识直接转化为实际交际语言能力, 还是一 种语法教学方法。

以趋向补语为例可以设计关于进出房间、上下楼梯等情景对话。再如对“把”字句进行练习, 可以设计我的周末，要求学生做完诸如洗衣服、写作业、打扫房间等事项，使用“把”字句。 通过情景练习有助于学生更好地理掌握语法使用的方法以及理解语法的语用功能。

\section{六. 语法练习和布置作业}

课堂上语法练习和课后作业是对学习进行巩固和强化不可缺少的手段。当学生理解相关语 法点后, 离学生独立使用语法点还是有一段距离的。学生理解了并不意味着能正确的运用, 语言习惯有一定的顽固性, 如: “*我去在大学”, 这样一个句子不是纠正过一两次学生就能完 全说对, 需要不断反复提醒纠正。尤其是初级阶段的汉语学习者需要多练, 注重反复。我注 意到有的汉语教师在进行语法练习方面采取的是语法讲解后, 直接让学生自己举例子或者布 置课后作业造句。初级阶段学生由于词汇量较小加上语法本身有一定的抽象性, 很多学生在 课堂上举例子通常想了很长时间, 举的例子也有问题。例如学了一年的兴趣班课后作业用“了” 和“没有”造句。结果出现了很多病句和问题句: “*他住了上海”、“*他有了三件事”、“*两年前 我结婚了好男人”、“*我没有买八个苹果”、“*我没有去在餐厅”、“*我没有看一个电影”。解决 的办法是可以在给初级阶段学生做语法练习和布置课后作业时, 考虑学生的水平, 给一定词 汇, 根据教授的语法点内容对学生的句子进行加以限制, 让学生造出正确合理的句子。如: 布置助词“了”课后练习时, 可以给几个动词, 如“吃”、“睡觉”、“听音乐”等词语。或者给学生 一个语言环境, 如: 我的周末做了那些事。这样学生造出来的句子限制在一定的范围内, 正 确率会大大提升, 有助于学生更好的掌握相关语法点。

\section{结语}

以上是本人从汉语初级阶段语法学习者偏误出发, 根据汉语语法自身的特点提出采用语言 对比法、简化浅化法、语义语用分析法、结构法、情境法等教学对策。汉语语法教学方法有 很多, 有的方法可应对多种语法教学, 一种语法也可以采取不同的教学方法或者采取多种方 法。至于初级阶段语法讲多少、讲到何种程度还要根据学生的实际情况而定。所以在实际教 学中, 汉语教师应当不拘泥于教材上的语法解释, 应该根据实际的教学情况对语法点重新加 工, 来提高教学效果。 


\section{参考文献}

Алещанова И. В., Фролова Н.А. К вопросу о способах формирования социальной компетенции на занятиях по иностранному языку // Совр. проблемы науки и образования (эл. изд.). 2015.№ 2-1. URL: http://www.science-education.ru /ru/article/view?id=20576 (дата обращения 23.01.2018).

黄伯荣、廖序东。现代汉语。北京：高等教育出版社，2007。下册。337页。

康玉华。汉语会话 301 句。北京: 北京语言大学出版社, 2006。下册。223 页。

李德津、程美珍。外国人实用汉语语法。北京：北京语言大学出版社，2008。652页。

李珠、蒋丽萍。怎样教外国人汉语。北京：北京语言大学出版社，2008。283 页。

周健、陈群。语感培养模式。北京：外语教学与研究出版社，2011。162 页。

赵金铭。对外汉语教学概论。北京：商务印书馆出版，2004，516 页。

\section{Ван Чжунцзюнь}

Новосибирский государственный университет

ул. Пирогова, 1, Новосибирск, 630090, Россия

mex2011@126.com

\section{МИКРОМЕТОДЫ В ОБУЧЕНИИ ГРАММАТИКЕ КИТАЙСКОГО ЯЗЫКА КАК ИНОСТРАННОГО НА НАЧАЛЬНОМ ЭТАПЕ}

Основная цель преподавания китайского языка как иностранного заключается в том, чтобы способствовать развитию способности коммуникации с носителями языка. Посредством анализа грамматических ошибок, допущенных на начальном этапе изучения, в данной статье изложены микрометоды обучения основам китайского языка.

Подробно исследуются вопросы грамматики учебных материалов 1-го и 2-го томов «Нового практического курса китайского языка» и учебника «Разговорная китайская речь» (в 2 ч.). Грамматические ошибки, допущенные русскими студентами в процессе изучения грамматики, проявляются в двух аспектах: использование служебных слов и порядок слов в предложении. Ошибки русских студентов делятся на четыре вида: пропуск, немотивированное добавление, замена иероглифа и ошибка в порядке слов.

В статье рассматриваются следующие микрометоды: сопоставление языков, упрощения, семантического анализа, структурный, ситуационный и т. д.

Метод сопоставления языков можно разделить на два типа: сопоставление китайского и иностранного, сопоставление внутри китайского языка. На начальной ступени в обучении грамматике китайского языка преподавателю следует использовать самые простые конструкции, чтобы помочь студенту их понять. Можно использовать два вида грамматических структур: фразы и предложения. При использовании приёма языковой ситуации необходимо 
соединить знание грамматики и реальное употребление языка. В практике обучения преподаватели китайского языка не должны однозначно придерживаться грамматических объяснений в учебных материалах. Им следует внедрять различные методы в соответствии с реальными условиями обучения, дорабатывать грамматические объяснения с целью повысить эффективность занятий.

Ключевые слова: преподавание китайского языка, начальный этап, изучение грамматики, микрометоды.

\section{Wang Zhongjun}

Novosibirsk State University

1 Pirogov Str., Novosibirsk, 630090, Russian Federation

mex2011@126.com

\section{ON THE MICRO SKILLS OF THE ELEMENTARY CHINESE GRAMMAR TEACHING IN THE COURSE OF CHINESE AS FOREIGN LANGUAGE}

The main aim of teaching Chinese as foreign language is to foster capability of social communication with Chinese native speakers. Through analyzing grammatical errors in the period of elementary Chinese studies, this paper expounds the micro skills for better teaching.

The involved grammar points in this paper were taken from «New Practical Chinese Reader Textbook» (vol. 1 and 2) and «Conversational Chinese 301» (in two parts). The grammatical errors are mainly revealed in two aspects: function word and words' order. We found that Russian students' grammatical errors can be divided into four categories: omission, improper addition, over representation and words' disorder. This paper presents some micro skills in teaching Chinese such as contrast method, simplification method, semantic and structure analysis, situational method etc.

Language contrast has two categories, the first is contrast between Chinese and another languages, the second is contrast within Chinese language. It is quiet difficult to learn Chinese grammar at the beginning period. Thus, the teachers should use simple constructions to explain Chinese grammar for students more easy understanding. In practical teaching we often use phrase and sentence structure to study Chinese grammar. We put the grammar in some contextual language environment by adopting situational method, then students can transfer grammar into practical communicative capacity, combining with semantic pragmatic analysis. The teachers of Chinese should flexibly adopt various methods, instead of rigidly adhering to the textbook, to promote efficiency in the beginning period of Chinese grammar teaching.

Key words: teaching Chinese, elementary course, grammar studies; micro skills.

\section{References}

Aleshchanova I. V., Frolova N. A. K voprosu o sposobakh formirovaniya sotsial'noi kompetentsii na zanyatiyakh po inostrannomu yazyku [On the problem of social competence formation's methods at the classes of Foreign languages]. Sovr. problemy nauki $i$ obrazovaniya (el. izdanie) 
[Contemporary problems of science \& education (electronic edition], 2015, № 2-1. URL: http://www.science-education.ru/ru/ article/view?id=20576 (accessed 23.01.2018). (in Russ.)

Huang Borong, Liao Xudong. Xiandai hanyu [黄伯荣、廖序东。现代汉语]. Modern Chinese. Beijing, Higher Education Press, 2007, vol. 2, 337 p. (in Chin.)

Kang Yuhua. Hanyu huihua 301 ju [康玉华。汉语会话 301 句] Conversational Chinese in 301 Phrases. Vol. 2, 223 p. (in Chin.)

Li Dejin, Cheng Meizhen. Waiguo ren shiyong hanyu yufa [李德津、程美珍。外国人实用汉语 语法]. A Practical Chinese Grammar for Foreigners. Beijing, Beijing Language and Culture University Press, 2008, 652 p. (in Chin.)

Li Zhu, Jiang Liping. Zenmeyang jiao waiguo ren hanyu [李珠、蒋丽萍。怎样教外国人汉语]. How to Teaching Chinese to Foreigners. Beijing, Beijing Language and Culture University Press, 2008, 283 p. (in Chin.)

Zhoujian, Chenqun. Yugan peiyang moshi [周健、陈群。语感培养模式]. The Model of Language Sense. Beijing, Language and Teaching Research Press, 2011, 162 p. (in Chin.)

Zhao Jinming. Duiwai hanyu jiaoxue gailun [赵金铭。对外汉语教学概论]. Introduction of Teaching Chinese as a Foreign Language. Beijing, The Commercial Press, 2004, 516 p. (in Chin.) 